\title{
NUMERICAL EXPERIMENTS ON THE TENSOR VIRIAL \\ IN $N$-BODY SYSTEMS
}

\author{
R. H. MILLER \\ Dept. of Astronomy, University of Chicago, Chicago, Ill., U.S.A.
}

\begin{abstract}
Tensor generalizations of the virial theorem were checked in a 100-body integration. The virial theorem was remarkably well satisfied, and the calculation confirmed the generalized Lagrange-Jacobi identities. The potential energy tensor, the kinetic energy tensor, and the virial tensor showed surprisingly long correlation times of about $\frac{1}{5}$ of a crossing time.
\end{abstract}

\section{Introduction}

The tensor generalization of the Lagrange-Jacobi identities for self-gravitating $n$-body systems (Chandrasekhar, 1964) can provide an independent check on $n$-body calculations beyond that provided by the usual first integrals of motion, even though it leads to no new constants of motion. These equations read:

$$
\frac{1}{2} \frac{\mathrm{d}^{2} I_{i j}}{\mathrm{~d} t^{2}}=2 T_{i j}+W_{i j}=V_{i j}
$$

where $I_{i j}$ is usually called the 'moment of inertia tensor' (it is not the same as the object called by that name in the mechanics books; cf. Goldstein, 1960), $T_{i j}$ is the kinetic energy tensor, and $W_{i j}$ is the potential energy tensor. The tensor that appears on the right hand side of Equation (1) is called $V_{i j}$ for convenience. These tensors are defined by

$$
\begin{aligned}
& I_{i j}=\sum_{\alpha=1}^{n} m_{\alpha} x_{i}^{(\alpha)} x_{j}^{(\alpha)}, \\
& T_{i j}=\frac{1}{2} \sum_{\alpha=1}^{n} m_{\alpha} \dot{x}_{i}^{(\alpha)} \dot{x}_{j}^{(\alpha)},
\end{aligned}
$$

and

$$
W_{i j}=-G \sum_{\alpha=1}^{n-1} m_{\alpha} \sum_{\beta=\alpha+1}^{n} m_{\beta} \frac{\left(x_{i}^{(\alpha)}-x_{i}^{(\beta)}\right)\left(x_{j}^{(\alpha)}-x_{j}^{(\beta)}\right)}{r_{\alpha \beta}^{3}} .
$$

Here, $\alpha$ and $\beta$ are indices that identify particles $(\alpha, \beta=1,2, \ldots, n), x_{i}^{(\alpha)}$ is the $i$-component of the position vector of particle $\alpha, \dot{x}_{i}^{(\alpha)}$ is its velocity, $m_{\alpha}$ is its mass, and $r_{\alpha \beta}$ is the (scalar) distance between particles $\alpha$ and $\beta$. Each of these tensors is manifestly symmetrical; the traces of $T_{i j}$ and of $W_{i j}$ are the usual (total) kinetic and potential energy of the cluster and the trace of Equation (1) is the usual Lagrange-Jacobi identity (Chandrasekhar, 1960).

Several remarks are in order concerning this system of equations. First, Equation(1) is a set of six independent ordinary differential equations concerning integral proper- 
ties of the system which follow directly from the equations of motion as an identity; they are not an approximation. The more familiar 'virial theorem', which follows from the assertion that the time-average of the left-hand side of Equation (1) should approach zero if the system is in a 'dynamically steady state', is, by contrast, a severe approximation. (For an illuminating discussion of the approximation from a mathematical point of view, see Pollard, 1966). Some comments on the apparent validity of these approximations appear later in this note.

Second, the fact that Equation (1) is a set of ordinary differential equations means that it does not lend itself to a check on $n$-body calculations as directly as do the usual ten first integrals (total energy, total angular momentum, and so on) because it does not lead to conserved quantities that lend themselves to simple checking or to use as controls on the integration (Miller, 1971; Nacozy, 1971). Rather, they require either another numerical integration or a numerical differentiation. Either of these procedures introduces its own difficulties into the overall numerical integration of the $n$ body system. Similarly, the "virial theorem" (and its tensor generalizations) yields, at best, a crude a posteriori check on the integration because the right-hand side of Equation (1) can depart substantially from zero at any instant.

Third, computation of $W_{i j}$ entails summation over pairs of particles, and thus tends to be expensive in calculations with reasonable numbers of particles. In addition, the quantities $W_{i j}$ and $T_{i j}$ vary substantially on the time scale of encounters between individual pairs of particles, so integration of the system (1) requires time-steps as fine as the shortest time-steps in use for any star of the cluster. This feature, coupled with the cost of determining $W_{i j}$, militates against using the tensor generalization of the Lagrange-Jacobi identities as a check or control on the integration.

Nonetheless, the 'tensor virial' equations can provide some insights into the behavior of star clusters, and thus are interesting on their own merits quite apart from any possible utility in providing an additional check on the integration. For example, they promise to provide a useful tool in the study of strongly rotating systems, which are expected to depart significantly from spherical symmetry. The studies reported here were not based on a strongly rotating system; the most interesting results relate to the correlation times that emerged in attempts to verify that the system obeys Equation (1).

\section{The $\boldsymbol{N}$-Body Integration}

These experiments made use of a data file generated for use as a classroom exercise for a course in stellar dynamics; while this file was a convenient source of data, it was somewhat restrictive in that it was not convenient to reconstruct features not already present in the file and because the file was designed for other purposes. The $n$-body calculation was patterned after that of Wielen (1967); a 100-body system was integrated for about 8 crossing-times. All particle masses were equal $(G=m=1)$, and a low value of total energy was chosen to scale the time-steps conveniently $(E=-26.4$, so $T_{\mathrm{cr}}$ is about 260 ; energy was conserved to about $0.3 \%$ over the entire run). The initial 
condition was generated by a Monte Carlo process to simulate a Plummer model. It had nonvanishing, but small, angular momentum; merely that left over because the Monte Carlo stopped after loading 100 particles instead of going on forever. The systematic rotation represented about $0.25 \%$ of the total kinetic energy at the beginning of the calculation. It was purposely left nonzero for the classroom exercises for which this run was designed. The data file consists of a set of 42 'snapshots' each giving the instantaneous coordinates and velocities for each of the 100 particles. These snapshots were made at equal intervals of time, $\Delta t=50$, or about five per crossing-time, covering the interval $t=0$ to $t=2050$.

The classroom exercises included (a) verification of the usual first integrals as checks on the calculation, (b) plots of some particle trajectories, (c) follow the changes in radial density distribution (watch for development of a 'core-halo' structure), (d) checks for formation of long-lived binaries, (e) checks for escaping particles, (f) determination of scatter in cluster mass estimates by the usual scalar virial theorem, making use of only the radial velocities and projected positions as seen with real clusters, and (g) some simple checks on the tensor virial relations. No strongly bound long-lived binaries were found, and two stars escaped from the cluster, but had not gone more than 3 cluster radii away by the end of the calculation at 8 crossing-times.

The results reported here were obtained in a more thorough study than was attempted in the student exercises. For each snapshot, the tensors $T_{i j}, W_{i j}, I_{i j}$, and $V_{i j}$ of Equations (2)-(4) were computed; the tensor elements were also averaged over the set of snapshots. Further, the second differences of elements of the tensor $I_{i j}$ (from successive snapshots) were computed as numerical approximations to the second time derivatives that appear in Equation (1).

\section{The Tensors}

The tensors $T_{i j}$ and $W_{i j}$, evaluated at each snapshot, were nearly diagonal and the diagonal elements of each tensor were nearly equal. There was considerable scatter in the individual values for the elements of each tensor. The diagonal elements of $V_{i j}$ were usually small - noticeably smaller than typical elements of either $2 T_{i j}$ or of $W_{i j}$, and not significantly larger than the off-diagonal elements. Although $V_{i j}$ was diagonalized at each snapshot, the directions of its principal axes jumped round so much that no pattern emerged. The elements of $I_{i j}$ grew to larger magnitude during the run; the diagonal elements were typically about 10 times as large as the off-diagonal elements.

The arithmetic-mean tensors averaged over all 42 snapshots showed a little more structure. Again, $T_{i j}$ and $W_{i j}$ were nearly diagonal (off-diagonal elements about $\frac{1}{30}$ of the diagonal elements), with the diagonal elements nearly equal. The scatter of each of these tensor elements, as evaluated for each snapshot, can be taken as some indication of the statistical accuracy with which the mean values are determined. On this basis, the diagonal elements of $T_{i j}$ and $W_{i j}$ were nearly equal (within 2 standard deviations); in both, the 33-component was about $10-13 \%$ larger than the 11 or 22 elements. Similarly, the mean off-diagonal elements are about 1.5-2 standard devia- 
tions away from zero. In view of the expected irregularity of these tensor elements, these tensors cannot convincingly be argued to be other than multiples of an identity matrix.

The arithmetic mean of the virial tensor, $V_{i j}$, yielded no element that was significantly nonzero by the same kind of statistical test. The fairly rapid convergence of the arithmetic-mean of the virial tensor to zero provides experimental evidence for the operation of a 'tensor virial theorem'; the left hand side of Equation (1) can be replaced by zero to a remarkably good approximation.

It is unfortunate that there does not seem to be a natural way to estimate the covariances of these tensor elements by appeal to higher order tensors constructed along the lines of $T_{i j}, W_{i j}$, or $I_{i j}$. A theory indicating how this might be done, and how the magnitudes of the elements might be estimated from integral cluster properties, relating the whole picture to first principles, should be interesting.
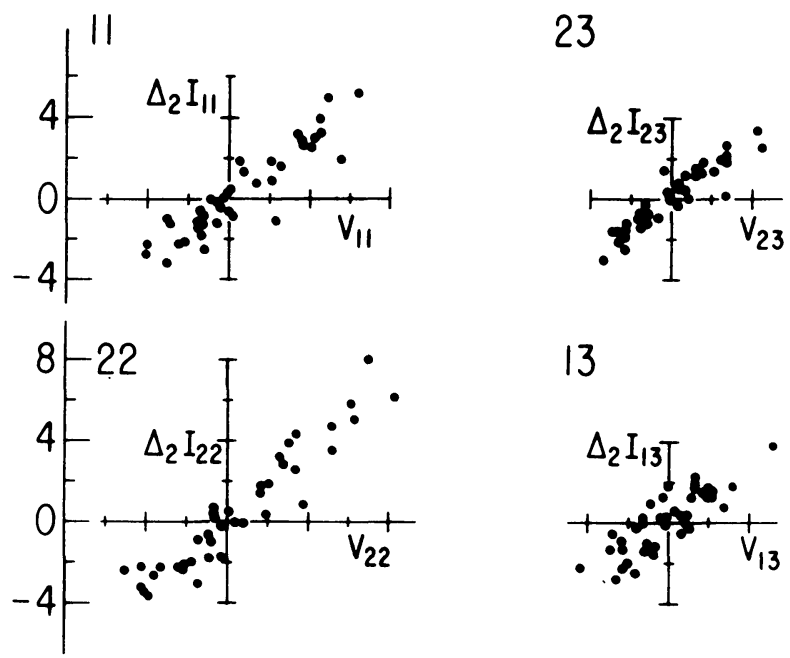

13
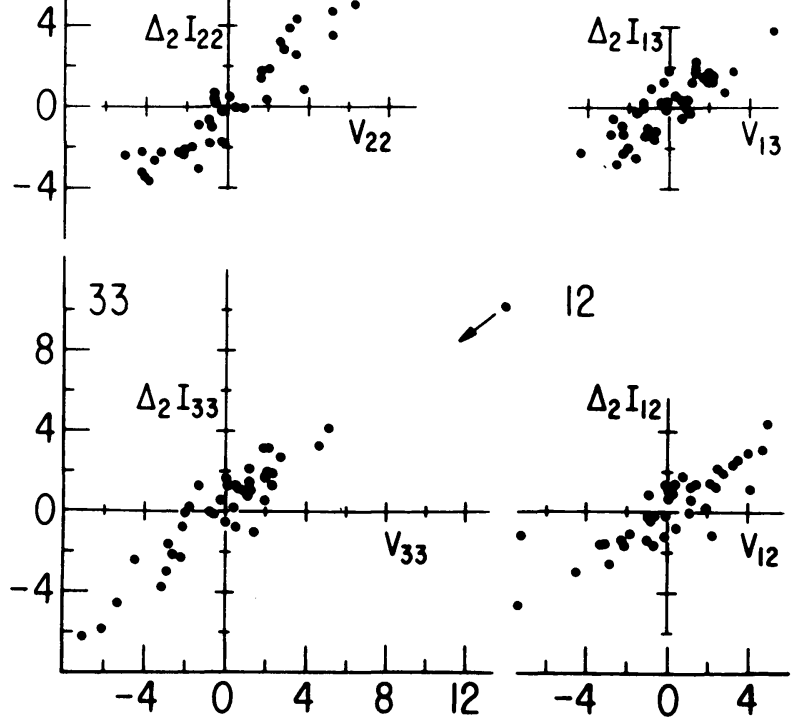

12

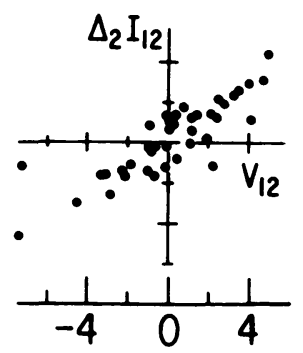

Fig. 1. Experimental checks on the identities of Equation (1). Each point represents one snapshot of the evolution of the 100-body system, with ordinate $\left(I_{i j}(s+1)+I_{i j}(s-1)-2 I_{i j}(s)\right) / 5000$, and abscissa $V_{i j}(s)$, where $s$ is the 'snapshot number', $(s=2,3, \ldots, 41)$. All points should lie on a straight line of unit slope through the origin. The scatter results from infrequent sampling, so the basic picture is confirmed. 


\section{The Identities}

As argued earlier, direct integration of Equation (1) is impractical. Both $T_{i j}$ and $W_{i j}$ vary on the time scale of individual encounters, so accurate integration would require use of time-steps as small as the shortest in use anywhere in the system. This would require very frequent evaluation of $W_{i j}$, which is expensive because it involves sums over pairs of particles. Samplings at $\frac{1}{5}$ of a crossing-time are nowhere nearly frequent enough to permit reliable integration of Equation (1).

The identities may be investigated as a differential relation, rather than by integration. The most convenient way to do this numerically is to compare values of the second differences of $I_{i j}$ with values of $V_{i j}$. A plot of half the second difference of $I_{i j}$ at time $t$ (suitably scaled to allow for the sampling interval) against $V_{i j}$ for the same time $t$, should yield a set of points that lie on a straight line through the origin with unit slope. Such a plot was made for each of the 6 independent tensor elements; but the points did not all lie on the line (Figure 1) rather, they scattered about the line. The underlying line is clearly discernable, but the scatter of points is appreciable. Evidently the sampled value of $V_{i j}$ need not accurately represent the average value over the sampling interval; similar changes can occur in $I_{i j}$. The second difference is notoriously difficult to obtain numerically in any case.

The scatter of points indicates that the sampling interval is not close enough to permit precise testing of Equation(1), but the appearance of the line confirms the basic picture. This is not simply a matter of everything being correlated with everything - a plot of the second difference of $I_{22}$ vs $V_{33}$, for example, gives a fairly complete scatter diagram. We conclude that the sampling interval used in these experiments is adequate to confirm the basic trend, but insufficient to show exact agreement. The evidence of time-scales is perhaps the most interesting result of these experiments; a fuller discussion of this point follows.

\section{Correlation Time}

The arithmetic means used in Section 3 are easily evaluated and thus represent the most natural experimental approach to the study of the 'virial theorem'. But they are neither a proper time-average nor a proper ensemble-average:

$$
\left\langle T_{i j}\right\rangle=\frac{1}{42} \sum_{s=1}^{42} T_{i j}(s \Delta t) \neq \frac{1}{42 \Delta t} \int_{0}^{42 \Delta t} \mathrm{~d} \tau T_{i j}(\tau) \neq \operatorname{Lim}_{\tau \rightarrow \infty} \frac{1}{\mathbb{T}} \int_{0}^{\pi} \mathrm{d} \tau T_{i j}(\tau) .
$$

The key to this question is the correlation time for typical changes in the relevant quantities. If the correlation time is at least on the order of $\Delta t$ (but significantly shorter than $42 \Delta t$ - the duration of the experiment), then the arithmetic mean approximates a time-average (over the restricted time available to the experiment; the 'significantly shorter than $42 \Delta t^{\prime}$ is required to approximate the arbitrarily long time interval in the definition of a time-average). On the other hand, if the correlation-time is much 
shorter than $\Delta t$, successive samples are statistically independent and the arithmetic mean approximates an ensemble average (again, with the proviso that 42 samples is hardly the very large number imagined in the definition of an ensemble average). A fundamental difficulty is that there is no a priori way to estimate the correlation-time for these quantities from first principles. Some attempts have been made along these lines for typical statistical-mechanical systems by Lebowitz et al. (1967) and by Zwanzig and Ailawadi (1969); however even for these simpler systems, general rules for computing correlation times for arbitrary quantities are not available.

The best recourse again seems to be experimental. Correlation times were studied through lagged products of the tensor elements, thus forming lagged autocorrelations. In all cases, the autocorrelation at lag 1 was $30-40 \%$ of that at lag 0 (the variance), and that at lag 2 or greater was essentially zero. Thus, experimentally, the correlation time for elements of $V_{i j}$ and for off-diagonal elements of $T_{i j}$ and $W_{i j}$ is about $\Delta t=50$, or $\frac{1}{5}$ of a crossing-time. Changes in the autocorrelation were masked by the large mean values for the diagonal elements of $T_{i j}$ and of $W_{i j}$.

It was rather surprising that so long a correlation-time appeared experimentally in view of the pathological variations in kinetic and potential energies noted in most integrations of $n$-body systems (see, for example, Wielen, 1967). Both $\left|T_{i j}\right|$ and $\left|W_{i j}\right|$ increase sharply as a pair of stars enters a close encounter. Because of the large number of close encounters that can occur, each of these quantities can vary quite strongly and quite rapidly. The strong encounters are of short duration, and a cluster looked at at some arbitrary time is not likely to be undergoing a particularly strong encounter. They also involve only two particles, typically, leaving the slower evolution of the entire system that dominates the longer time structure. This allows the weaker longlag autocorrelation to appear, but loses a significant short-lag contribution.

The comparison of second differences of $I_{i j}$ with $V_{i j}$ discussed in Section 4 involves similar considerations of correlation time. If there were rapid variations in either, the comparison should not work out with this kind of sampling. The scatter of points away from the line $\frac{1}{2} \ddot{I}_{i j}=V_{i j}$ indicates that some detail has been lost. But some information survives even this infrequent sampling, indicating that the correlation time for the second difference of $I_{i j}$ and for $V_{i j}$ is on the order of the sampling interval. Lagged plots, like those of Figure 1 in which the points are located by the second difference of $I_{i j}$ at snapshot number $s$ and by $V_{i j}$ at snapshot number $(s+1)$, show barely discernable traces of the line. This, too, implies that the correlation time for values of these tensor elements is about one sampling interval, but does not extend over twice the sampling interval.

\section{Discussion and Conclusions}

The elements of the virial tensor, $V_{i j}$, fluctuated rapidly with mean zero and standard deviation about $2 \%$ of the cluster energy for this 100 -body system. Presumably larger fractional variations would occur with fewer bodies. The tensor generalization of the virial theorem was surprisingly well confirmed for this system. 
Correlation times for rapidly varying quantities, with the kinetic energy tensor, the potential energy tensor, and the virial tensor as prototypes, were about $\frac{1}{5}$ of a crossingtime for this system. The correlation times describe the dominant time-scale of fluctuations of these quantities, and probably give some measure of the time-scale of phase-mixing.

The identities of Equation (1) do not provide a useful check for $n$-body integrations because they are expensive to evaluate and difficult to use. However, it has been confirmed that the integration generates a system that obeys these identities.

The interpretations given in this note are subject to the usual difficulties that attend numerical experiments with self-gravitating $n$-body systems. These arise from the errors of numerical integration and from a tendency to overinterpret results because one knows too much about what is going on in the system. Both sets of problems are best handled by using several samples of data - calculations run from different sets of initial conditions to sample the parameter space of initial conditions. That recourse was not available for this experiment for economic reasons, so other arguments are required.

The numerical integration errors can be disposed of because the time scales of the quantities studied in this experiment are not long compared to the $e$-folding time for error growth; however, those time scales might be of the same order of magnitude. The dangers of over-interpreting one experiment are not troublesome here because the experiment was undertaken to check on some calculation details in a sample integration. As more general statements are made, the position becomes more precarious. The conclusions about correlation time may be affected by the fact that there were no very close encounters in this system. But a close encounter in which the potential energy of one star pair is equal to the potential energy of all the rest of the cluster must occur much less frequently with 100 bodies than with 16 . This system may be special, but there is no strong reason to believe that it is; at any rate, this is a danger that affects all experimentation. We conclude that the results presented here withstand scrutiny on both counts.

\section{References}

Aarseth, S. J.: 1971, Astrophys. Space Sci. 14, 118.

Chandrasekhar, S. : 1960, Principles of Stellar Dynamics, Dover Publications Inc., New-York.

Chandrasekhar, S.: 1964, in W. E. Brittin (ed.), Lectures in Theoretical Physics, University of Colorado Press, Boulder, Colo., Vol. 6, p. 1.

Goldstein, H.: 1960, Classical Mechanics, Addison-Wesley, Cambridge, Mass.

Hoerner, S. von: 1960, Z. Astrophys. 50, 184.

Lebowitz, J. L., Percus, J. K., and Verlet, L.: 1967, Phys. Rev. 153, 250.

Miller, R. H.: 1971, J. Comput. Phys. 8, 464.

Nacozy, P. E.: 1971, Astrophys. Space Sci. 14, 40.

Pollard, H.: 1966, Mathematical Introduction to Celestial Mechanics, Prentice Hall Inc., Englewood Cliffs, N.J.

Wielen, R.: 1967, Veröffentl. Astron. Rechen-Inst. Heidelberg, Nr. 19.

Zwanzig, R. and Ailawadi, N. K.: 1969, Phys. Rev. 182, 280. 


\section{DISCUSSION}

King: Your mention of ensemble averaging raises a serious general problem: how do we extract meaningful distribution functions from a small number of bodies in a simulation? Clearly the answer is in the averaging of snapshots, as you have suggested; but I think that the general statistical problem is one on which we need serious new work.

Miller: One of the usual pitfalls is a tendency to take snapshots at a fixed number of integration steps, which makes it more likely to take the snapshot during a close encounter. This leads to a strong bias in sampled quantities, which is, however, well-known to those who do such $n$-body integrations. 